\title{
Mananoligossacarídeo em dietas para larvas de tilápia
}

\author{
Kátia Kalko Schwarz ${ }^{1}$, Wilson Massamitu Furuya ${ }^{2}$, Maria Raquel Marçal Natali ${ }^{3}$, Mirian \\ Cristina Gaudezi ${ }^{4}$, Patrícia Alves Gonçalves de Lima ${ }^{4}$
}

\footnotetext{
1 Universidade Estadual de Maringá, Pós Graduação em Zootecnia/PPZ-UEM/Faculdade Estadual de Filosofia, Ciências e Letras de Paranaguá/FAFIPAR.

2 Universidade Estadual de Maringá, Departamento de Zootecnia, Programa de Pós-Graduação em Zootecnia UEM/PPZ.

3 Universidade Estadual de Maringá, Departamento de Biologia, UEM.

${ }^{4}$ Graduanda do Curso de Ciências Biológicas da Faculdade Estadual de Filosofia, Ciências e Letras de Paranaguá/FAFIPAR.
}

RESUMO - Este experimento foi realizado para avaliar níveis crescentes de mananoligossacarídeo (MOS) na dieta de larvas de tilápias-do-nilo (linhagem Supreme ${ }^{\circledR}$ ) na fase de reversão sexual durante 30 dias. Larvas de tilápia-do-nilo ( $\mathrm{n}=1500$, $\mathrm{P}=0,01 \mathrm{~g} \pm 0,001 \mathrm{~g}$ ) foram distribuídas aleatoriamente em 30 tanques de 100 litros. Os peixes foram distribuídos em delineamento inteiramente casualizado, composto por seis tratamentos e cinco repetições. A dieta controle (isenta de MOS) foi elaborada para conter aproximadamente $35 \%$ de proteína bruta e $3.100 \mathrm{kcal} \mathrm{de} \mathrm{ED} / \mathrm{kg}$. As demais foram suplementadas com $0 ; 0,15 ; 0,30 ; 0,45 ; 0,60$ e $0,75 \%$ de MOS em substituição ao milho da dieta controle. Com o aumento nos níveis de MOS nas dietas, foi observado aumento linear sobre o comprimento do intestino, a altura das vilosidades intestinais e a densidade dos vilos. Não houve diferença na composição corporal, no ganho em peso, no peso final, no comprimento final, no fator de condição, na taxa de sobrevivência e no número de células caliciformes do intestino das larvas. Os níveis de MOS tiveram efeito quadrático sobre a conversão alimentar, cujos melhores resultados foram obtidos com $0,34 \%$ de MOS. A utilização de MOS no nível de $0,34 \%$ em dietas para larvas de tilápia-do-nilo melhora a conversão alimentar e promove aumento do comprimento do intestino, da altura das vilosidades e da densidade de vilos intestinal.

Palavras-chave: desempenho, mucosa intestinal, peixe, prebiótico

\section{Mannanoligosaccharides in diets for tilapia larvae}

\begin{abstract}
This experiment was conducted to evaluate increasing levels of mannanoligosaccharides (MOS) in the diet of Nile tilapia larvae $\left(\right.$ Supreme $\left.^{\circledR}\right)$ at the sex reversal stage for 30 days. Nile tilapia larvae $(n=1500, p=0.01 \pm 0.001 \mathrm{~g})$, were randomly distributed in 30 tanks of 100 liters each, for 30 days. The fish were distributed in a randomized design with six treatments and five replicates. The control diet (no MOS) was elaborated to contain approximately $35 \%$ of crude protein and $3,100 \mathrm{kcal} \mathrm{DE} / \mathrm{kg}$. Mannanoligosaccharides were included in the other diets, replacing corn, at levels of $0,0.15,0.30,0.45$, 0.60 or $0.75 \%$. Linear increase in the intestine length, intestinal villous height and villi density was observed with the dietary MOS increase. No differences in body composition, weight gain, final weight, final length, condition factor, survival rate or number of goblet cells of the gut of the larvae were observed. A quadratic effect on feed conversion ratio was observed with the MOS inclusion and the best value was estimated with $0.34 \%$. Dietary MOS inclusion at $0.34 \%$ improves feed conversion and increases intestine length, villi height and density of intestinal villi in diets for Nile tilapia larvae.
\end{abstract}

Key Words: fish, intestinal morphology, performance, prebiotic

\section{Introdução}

No Brasil, tilápia-do-nilo (Oreochromis niloticus) é a espécie exótica mais criada em tanques-rede. Em 2004, a produção de tilápias no Brasil foi de $26 \%$ do total produzido na aquicultura nacional, e o país respondeu por $64 \%$ da produção total desta espécie de peixe (Ostrensky et al., 2008).

Por apresentar excelentes índices produtivos, o cultivo de tilápias ainda necessita de estudos quanto à sua nutrição, principalmente quanto à utilização de aditivos para melhoria do desempenho. Com a restrição do uso de alguns promotores de crescimento, principalmente os antibióticos e alguns quimioterápicos, o uso de mananoligossacarídeos (MOS) nas rações tem promovido resultados interessantes no desempenho desta espécie.

O mananoligossacarídeo (MOS) proveniente da parede celular da levedura Sacharomyces cerevisiae tem sido utilizado em dietas com o objetivo de melhorar a conversão alimentar, a integridade da mucosa intestinal e a saúde dos peixes, resultando em melhor ganho econômico 
(Li \& Gatlin III, 2004). É um prebiótico que possui na sua composição química cerca de $40 \%$ de $\beta$-glucanos, $40 \%$ de $\alpha$-mananos, $28 \%$ de proteínas, $7 \%$ de lipídeos, $3 \%$ de substâncias inorgânicas e $2 \%$ de hexosaminas e quitina. O glucano é o componente estrutural mais abundante e está localizado na parte interna da parede, enquanto o manano localiza-se na parte externa (Hough, 1990).

A utilização desses polissacarídeos melhora a saúde de peixes (Robertsen et al., 1994; Sakai, 1999) e atua sobre o sistema imunológico e na prevenção da colonização de bactérias patogênicas no trato gastrintestinal do animal (Spring, 2001; Staykov et al., 2007; Refstie et al., 2010), podendo ter efeitos benéficos na morfologia intestinal (Yilmaz et al., 2007; Salze et al., 2008; Schwarz et al., 2010; Refstie et al., 2010). Além disso, melhora a utilização de alguns nutrientes (Hisano, 2005) e o desempenho produtivo dos peixes (Li \& Gatilin III, 2003; Culjak et al., 2006; Staykov et al., 2005; Staykov et al., 2007; Grisdale-Helland et al., 2008; Sado et al., 2008; Schwarz et al., 2009; Schwarz et al., 2010), mas há ainda poucas informações sobre a ação desses prebióticos sobre a morfologia intestinal de peixes, especialmente durante a masculinização, período em que a taxa de mortalidade é alta.

Este trabalho foi realizado com o objetivo de avaliar níveis crescentes de MOS em dietas para larvas de tilápia no período de masculinização e seus efeitos no desempenho produtivo, no comprimento intestinal, na morfometria das vilosidades intestinais e na contagem das células caliciformes.

\section{Material e Métodos}

Larvas de tilápia-do-nilo $(\mathrm{n}=1500 ; \mathrm{P}=0.01 \pm 0,001 \mathrm{~g})$, oriundas da piscicultura Aquabel - Rolândia, Paraná, foram distribuídas aleatoriamente em 30 tanques de 100 litros cada, no Laboratório de Nutrição de Peixes da Faculdade Estadual de Filosofia, Ciências e Letras de Paranaguá/ FAFIPAR, no período de novembro a dezembro de 2007, durante 30 dias. Os peixes foram distribuídos em delineamento inteiramente casualizado, com seis tratamentos e cinco repetições, considerando unidade experimental cada tanque de $100 \mathrm{~L}$ com 50 larvas.

A dieta controle foi elaborada para conter aproximadamente $35 \%$ de proteína bruta, $3.124 \mathrm{kcal}$ de ED/kg, de forma a atender às exigências recomendadas pelo NRC (1993) para as tilápias. Como prebiótico, o MOS utilizado foi o produto comercial SAF-Mannan ${ }^{\circledR}$ contendo $23 \%$ de $\beta$-glucano e $21 \%$ de $\alpha$-mananos, $28 \%$ PB, $1 \%$ P, $95 \%$ MS, $20 \%$ gordura e $4 \%$ de cinzas e foi incluído na proporção de
$0 ; 0,15 ; 0,30 ; 0,45 ; 0,60$ e $0,75 \%$ da dieta em substituição ao milho.

Todos os alimentos foram moídos em moinho martelo com peneira contendo orifícios de $0,5 \mathrm{~mm}$ de diâmetro. Após adição e homogeneização dos ingredientes, foi feita a inclusão de hormônio masculinizante. O hormônio $17 \alpha$-metiltestosterona foi diluído na proporção de $60 \mathrm{mg}$ em $400 \mathrm{~mL}$ de álcool $90^{\circ} \mathrm{GL}$ e adicionado à dieta, seco por 12 horas em temperatura ambiente, conforme recomendação de Hayashi (1995).

A dieta total foi distribuída, inicialmente, em seis refeições, às $8 \mathrm{~h}, 11 \mathrm{~h}, 14 \mathrm{~h}, 17 \mathrm{~h}, 20 \mathrm{~h} \mathrm{e} 23 \mathrm{~h}$. Posteriormente, após uma semana, as larvas receberam quatro refeições às $8 \mathrm{~h}, 12 \mathrm{~h}, 18 \mathrm{~h}$ e $23 \mathrm{~h}$, por meio de arraçoamento manual até saciedade aparente.

Diariamente, os tanques foram sifonados para retirada das fezes, sendo mantida a renovação de $10 \%$ do volume total de água de cada tanque. A temperatura foi mensurada diariamente pela manhã, às $8 \mathrm{~h}$, e no período da noite, às 23 h. $\mathrm{O}$ pH, nitrito, a amônia e o oxigênio dissolvido para acompanhamento de qualidade da água dos peixes foram medidos a cada três dias, com auxílio de kit digital portátil. O oxigênio dissolvido foi mantido entre $4,0 \mathrm{e}$ $6,0 \mathrm{mg} / \mathrm{L}$, por meio de pedra porosa acoplada a um soprador. A temperatura foi mantida entre 26 e $30^{\circ} \mathrm{C}$ por aquecedores de 80 Watts, todavia, como podem ocorrer alterações bruscas de temperatura durante o experimento, os aquecedores podem não manter a temperatura mínima de $26^{\circ} \mathrm{C}$ e este é um fator de desafio ambiental para a espécie de peixe estudada.

Ao final do experimento, em cada unidade experimental, três peixes foram retirados ao acaso, abatidos por secção medular, pesados e medidos quanto ao comprimento corporal e do intestino, sendo retirada a porção média para as análises das vilosidades intestinais de cada tratamento, totalizando 90 peixes abatidos e 15 por tratamento para esta análise.

No preparo do tecido para a análise microscópica, a porção média dos intestinos coletados foi mantida 24 horas em solução fixadora de alfaque ( $85 \%$ de álcool $85^{\circ}, 10 \%$ de formaldeído e $5 \%$ de ácido acético glacial), e o material foi transferido para solução em álcool $70 \%$ por mais 24 horas.

O processamento das lâminas histológicas foi realizado no Laboratório de Histotécnica Animal da Universidade Estadual de Maringá, conforme procedimento de rotina, sendo submetido à desidratação, por tratamento com álcool em concentrações crescentes (70 a 100\%). As amostras foram, então, diafanizadas e incluídas em parafina histológica. Os cortes foram realizados com sete micrômetros de espessura, cortados no sentido longitudinal, semisseriados 
e corados com hematoxilina e eosina (HE) e outro conjunto de lâminas coradas pelo processo de histoquímica Schiff o PAS (do inglês, Periodic acid-Schiff) por este reativo ter afinidade com as moléculas de glicogênio e proteoglicanas, presentes em células produtoras de muco, como as caliciformes, para sua melhor visualização.

Cada lâmina histológica continha cerca de 12 porções de tecido para análise. As imagens das lâminas histológicas foram capturadas com auxílio de microscópio ótico acoplado a uma câmara de captura de imagem, com computador contendo placa de TV ao sistema analisador de imagens Leica (Image-Pro Plus, versão 4.5.0.27).

Para as lâminas histológicas analisadas, foram escolhidos pelo critério de integridade seis tecidos por lâmina e por peixe. Foram medidas cinco vilosidades por tecido obtido por campo de captura de imagem, totalizando a leitura de 450 vilos por tratamento. Em seguida, foi realizada a captura de imagens por meio do software Ipwin32.ex/image-Pro Plus para mensuração da altura das vilosidades, em micrômetro e contagem de células caliciformes por vilo em aumento de 40 vezes. A densidade de vilos foi contada, de acordo com o campo de captura, ou seja, foram contadas todas as vilosidades contidas no campo de captura de imagem.

Todos os peixes foram pesados em balança analítica $(0,0001 \mathrm{~g})$ no início e no final do experimento, sendo o comprimento total obtido por meio de paquímetro digital eletrônico.

A taxa de eficiência proteica foi calculada de acordo com a expressão descrita por Jauncey \& Ross (1982). A composição química da carcaça foi obtida utilizando-se todos os peixes de cada unidade experimental com vísceras e escamas, excluindo os peixes utilizados para análise histológica. As análises bromatológicas foram realizadas conforme Silva \& Queiroz (2002) no Laboratório de Nutrição Animal/LANA na Universidade Estadual de Maringá/UEM.

As análises químicas das dietas e das carcaças foram realizadas no Laboratório de Análise de Alimentos do
Departamento de Alimentos da Universidade Estadual de Maringá-UEM, seguindo metodologia citada por Silva \& Queiroz(2006).

Os dados foram submetidos às análises de variância e regressão poligonal, por meio do programa SAEG (Sistemas de Análises Estatísticas e Genéticas) (UFV, 2000).

\section{Resultados e Discussão}

Os valores obtidos pelas variáveis físico-químicas da água dos tanques foram de 19 a $31{ }^{\circ} \mathrm{C}$, oxigênio dissolvido de 4,0 a $6,0 \mathrm{mg} / \mathrm{L}, \mathrm{pH}$ entre 6 e 7,5, amônia com valor médio de $0,25 \mathrm{mg} / \mathrm{L}$ e nitrato $0,50 \mathrm{mg} / \mathrm{L}$, e estes valores não diferiram entre os tratamentos. As alterações na qualidade da água foram ocasionadas pelas variações bruscas climáticas durante o período experimental.

Não foram observadas diferenças $(\mathrm{P}>0,05)$ no peso final, no ganho de peso, no comprimento final, no consumo de ração e na taxa de sobrevivência durante o processo de masculinização (Tabela 1).

Para a taxa de sobrevivência, considerando a variação de temperatura que ocorreu durante o experimento (19 a $31{ }^{\circ} \mathrm{C}$ ), os valores obtidos são importantes, pois um dos problemas enfrentados pelas empresas de larviculturas e das pisciculturas são as altas taxas de mortalidade no início do povoamento dos tanques e, entre outros fatores, a variação da temperatura é considerada um constante desafio em condições práticas.

De acordo com Tachibana et al. (2004), os valores de mortalidade obtidos são comuns durante a fase de masculinização em produções comerciais. Os dados de sobrevivência obtidos neste estudo aproximaram-se dos encontrados por Maracanas (1997), que observou taxa de sobrevivência de $62,5 \%$ para tilápias-do-nilo da linhagem tailandesa aproximando-se também dos $69 \%$ obtidos por Vera Cruz \& Mair (1994) para larvas da mesma espécie.

Foi observado efeito quadrático $(\mathrm{P}<0,05)$ da inclusão de MOS na dieta sobre a conversão alimentar, cujos melhores

Tabela 1 - Desempenho de larvas de tilápia-do-nilo na fase de reversão sexual alimentadas com dietas contendo mananoligossacarídeo

\begin{tabular}{lccccccc}
\hline Variável & \multicolumn{5}{c}{ Mananoligossacarídeo (\%) } \\
\cline { 2 - 7 } & 0 & 0,15 & 0,30 & 0,45 & 0,60 & 0,75 & $\mathrm{CV}^{1}$ \\
\hline Peso inicial (mg) & 0,01 & 0,01 & 0,01 & 0,01 & 0,01 & 0,01 \\
Peso final (mg) & 0,70 & 0,69 & 0,75 & 0,72 & 0,69 & 0,00 \\
Ganho em peso (mg) & 0,70 & 0,69 & 0,74 & 0,72 & 0,68 & 0,80 & 15,19 \\
Comprimento inicial (mm) & 0,07 & 0,07 & 0,07 & 0,07 & 0,07 & 15,31 \\
Comprimento final (mm) & 34,26 & 34,15 & 35,26 & 34,89 & 33,12 & 0,07 & $3,59,88$ \\
Conversão alimentar & 2,23 & 1,60 & 1,50 & 1,65 & 1,82 & 1,49 \\
Taxa de sobrevivência & 61,50 & 69,60 & 72,00 & 63,00 & 68,80 & 62,50 & 11,91 \\
\hline
\end{tabular}


valores foram obtidos com o nível de $0,34 \%$ de MOS. Esses resultados contrariam os encontrados por Yilmaz et al. (2007) em pesquisa com trutas (Oncorhynchus mykiss) na conversão alimentar. Por outro lado, os autores notaram diferenças nos demais parâmetros de desempenho dos peixes alimentados com a dieta contendo 1,5\% de MOS na dieta. Grisdale-Helland et al. (2008) também não encontraram diferenças no crescimento, na digestibilidade e na taxa de consumo de salmões (Salmo salar) alimentados com dietas contendo $0,1 \%$ de MOS, porém citam que o MOS e o FOS (frutoligossacarídeo) podem ser benéficos na produção de peixes desta espécie.

O melhor resultado de conversão alimentar encontrado neste estudo (Tabela 1) provavelmente está relacionado ao melhor aproveitamento das dietas, em razão do tempo de trânsito, da digestibilidade e da taxa de absorção dos nutrientes da dieta. Além disso, destaca-se na literatura a ação destes polissacarídeos em melhorar a saúde dos peixes (Robetsen et al., 1994; Sakai, 1999, Staykov et al., 2007), atuando sobre o sistema imunológico e na prevenção da colonização de bactérias patogênicas no trato gastrointestinal do animal (Spring, 2001; Refstile et al., 2010). Também por melhorar a utilização de alguns nutrientes (Hisano et al., 2008) e, consequentemente, o desempenho produtivo dos mesmos (Li \& Gatilin III, 2003; Schwarz et al., 2005; Schwarz et al., 2010).

Não houve diferença $(\mathrm{P}>0,05)$ nos teores de umidade, proteína, extrato etéreo, cinzas, cálcio e fósforo da carcaça dos peixes entre os níveis de MOS (Tabela 2).
Com o aumento nos níveis de inclusão de MOS nas dietas, foram observados efeitos quadráticos sobre os dados da altura de vilosidades intestinais e efeito linear sobre a densidade de vilos e o comprimento de intestino, não havendo diferença significativa no número de células caliciformes (Tabela 3 ).

Os resultados obtidos neste estudo estão de acordo com os descritos por Yilmaz et al. (2007), que encontraram melhor altura de vilosidades em trutas (Oncorhynchus mykiss) alimentadas com 1,5 e 3,0\% de MOS na dieta. Salze et al. (2008) trabalharam com bijupirá (Rachycentron canadum) em diferentes níveis de salinidade da água utilizando 0,2\% de MOS na dieta e observaram maior altura nas microvilosidades nos peixes que recebiam dieta com MOS. Schwarz et al. (2010) estudaram o efeito do MOS em juvenis de tilápia e observaram diferenças significativas nas alturas das vilosidades nos peixes que receberam $1 \%$ do MOS na dieta em comparação aos do tratamento testemunha.

O uso de MOS na dieta teve influência positiva na densidade de vilos e no comprimento do intestino, resultando em uma mucosa com maior integridade. Neste trabalho, os efeitos de MOS sobre o comprimento do intestino e a altura de vilosidades podem ser explicados pela redução da colonização de bactérias, porque atua inibindo sua aderência ao enterócito, por meio da ligação com o glicocalix. A exclusão competitiva também tem como princípio a aderência de bactérias não-patogênicas a sítios de ligação dos enterócitos (glicocalix) nos diferentes segmentos do trato gastrointestinal (TGI) (Furlan, 2005).

Tabela 2 - Composição química da carcaça de larvas de tilápia-do-nilo na fase de masculinização alimentadas com dietas contendo mananoligossacarídeo (valores expressos na matéria natural)

\begin{tabular}{|c|c|c|c|c|c|c|c|}
\hline \multirow[t]{2}{*}{ Variável } & \multicolumn{6}{|c|}{ Mananoligossacarídeo (\%) } & \multirow[b]{2}{*}{$\mathrm{CV}$} \\
\hline & 0 & 0,15 & 0,30 & 0,45 & 0,60 & 0,75 & \\
\hline Umidade & 82,99 & 8259 & 82,97 & 82,90 & 83,16 & 82,22 & 1,26 \\
\hline Proteína & 10,58 & 10,69 & 10,57 & 10,67 & 10,51 & 10,99 & 2,09 \\
\hline Extrato etéreo & 8,51 & 8,10 & 8,25 & 8,40 & 8,40 & 7,54 & 8,43 \\
\hline Cinzas & 2,47 & 2,68 & 2,67 & 2,54 & 2,58 & 2,82 & 2,73 \\
\hline Cálcio & 0,80 & 0,81 & 0,69 & 0,76 & 0,76 & 0,86 & 11,55 \\
\hline Fósforo & 0,51 & 0,63 & 0,55 & 0,61 & 0,60 & 0,51 & 9,22 \\
\hline
\end{tabular}

$\mathrm{CV}=$ ceficiente de variação.

Tabela 3 - Parâmetros morfométricos da porção média do intestino e comprimento do intestino de larvas de tilápias-do-nilo alimentadas com dietas contendo mananoligossacarídeo

\begin{tabular}{lccccccc}
\hline Parâmetro & \multicolumn{7}{c}{ Mananoligossacarídeo (\%) } \\
\cline { 2 - 6 } & 0 & 0,15 & 0,30 & 0,45 & 0,60 & 0,75 \\
\hline Altura do vilo $(\mu \mathrm{m})^{2}$ & 37,53 & 44,69 & 41,37 & 43,30 & 49,83 & 47,95 \\
Densidades de vilo ${ }^{2}$ & 7,60 & 8,13 & 9,00 & 9,55 & 9,61 & 16,18 \\
Células caliciformes/vilo & 6,34 & 7,64 & 8,01 & 7,67 & 7,19 & 9,35 \\
Comprimento intestino total $(\mathrm{mm})$ & 97,84 & 126,37 & 127,7 & 129,25 & 138,41 & 8,43 & 17,62 \\
\hline
\end{tabular}

$\mathrm{CV}=$ coeficiente de variação.

1 Efeito linear: altura de vilos: $\mathrm{Y}=39,1370+13,1030 \mathrm{X}: \mathrm{R}^{2}=0,68$; densidade de vilos: $\mathrm{Y}=7,872+2,71324 \mathrm{X}: \mathrm{R}^{2}=0,80 ;$ comprimento de intestino: $\mathrm{Y}=108,306+$ $47,9577 \mathrm{X} . \mathrm{R}^{2}=0,73$ 
Entretanto, conforme descrito por Gibson \& Roberfroid (1995), os prebióticos em geral não devem ser hidrolisados ou absorvidos no intestino delgado e normalmente atuam como substrato seletivo para determinado grupo de bactérias comensais benéficas, sendo capaz de alterar de forma positiva a microbiota intestinal. Assim, se o trato gastrintestinal está em condições saudáveis, a integridade da mucosa intestinal terá melhores condições de desenvolvimento e desempenho, resultando em uma mucosa com vilos íntegros, de tamanho e densidade maiores, proporcionando incremento do comprimento intestinal.
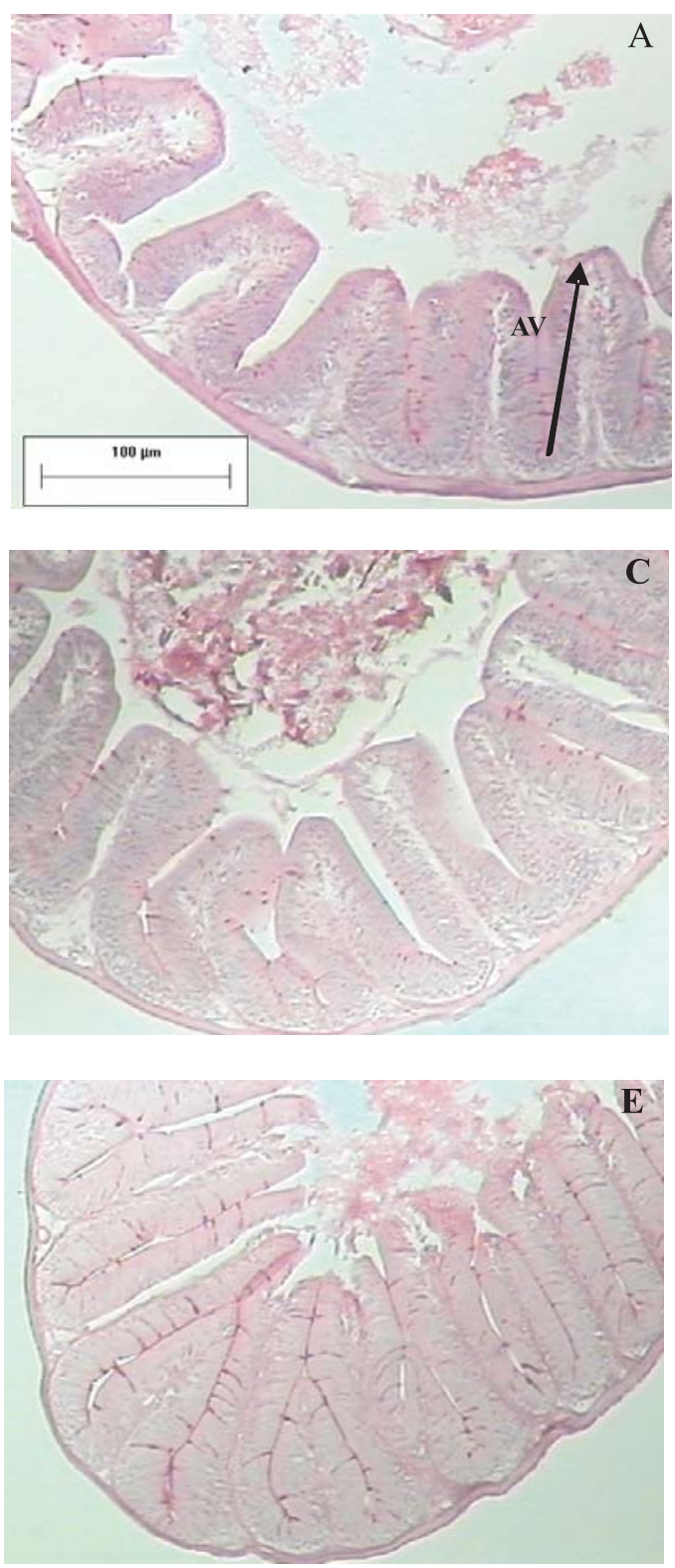

Os oligossacarídeos, presentes nestes prebióticos, possuem 2 até 10 unidades de monossacarídeos (Bacila, 2003). As cadeias de oligossacarídeos são processadas no complexo de Golgi e, nas células intestinais produtoras de muco (células caliciformes), teriam ligação com este processo de liberação de muco intestinal (Alberts et al., 2004; Junqueira \& Carneiro, 2005). Essa possibilidade pode ser um indício de que algumas partículas de oligossacarídeos, provindas dos prebióticos, podem ser absorvidas no trato gastrintestinal.

Outro fator de considerável relevância é que os açúcares presentes no MOS têm ligação com a formação
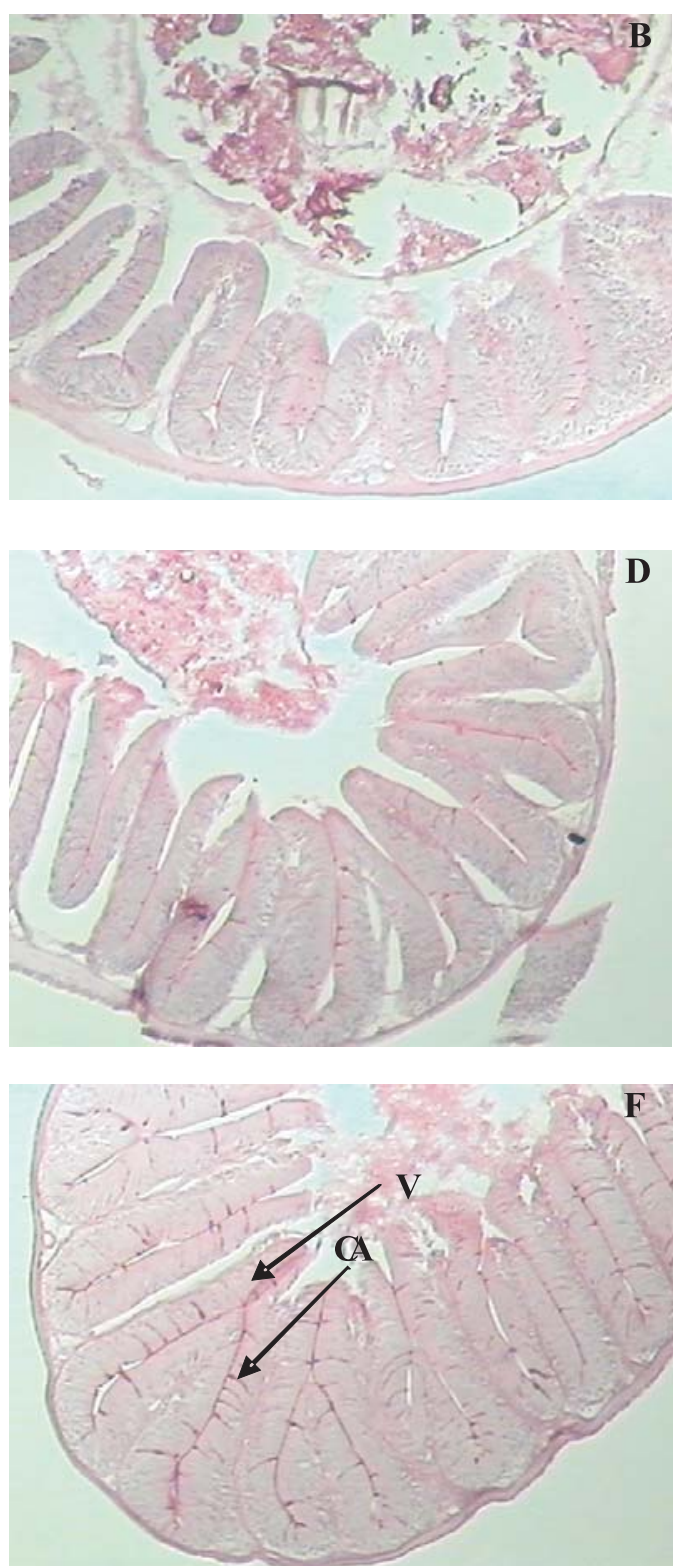

Observar as diferenças da densidade de vilos e da integridade do tecido intestinal. $\mathrm{A}=0 \% ; \mathrm{B}=0,15 \% ; \mathrm{C}=0,30 \% ; \mathrm{D}=0,45 \% ; \mathrm{E}=0,60 \%$; $\mathrm{F}=0,70 \%$ de $\mathrm{MOS}$ na dieta . $\mathrm{AV}=$ altura de vilosidade; $\mathrm{CA}=$ células caliciformes; $\mathrm{V}=$ vilosidades. Coloração: P.A.S. Barra $100 \mu \mathrm{m}$.

Figura 1 - Imagem de microscópio ótico acoplado ao sistema analisador de imagens Leica (Image-Pro Plus versão 4.5.0.27). 
e constituição com o citoesqueleto celular (Alberts et al., 2004) e estas, por sua vez, podem ter alguma influência na composição do tecido gastrintestinal, melhorando sua morfologia.

Essa ligação pode interferir na integridade da mucosa, principalmente dos enterócitos, na sua borda escova ou nas microvilosidades, o que confere à capacidade desta célula absortiva maior eficiência. Além disso, o posicionamento, conforme relatos de Furlan (2005), do glicocálix não apenas atua como um sistema de aderência da bactéria ao enterócito, mas pode armazenar e concentrar as enzimas digestivas produzidas pelas bactérias, que atuariam sobre a mucosa intestinal.

Junqueira \& Carneiro (2005) citaram que, quanto maior o tamanho da vilosidade intestinal, maior a capacidade de absorção de alimentos pelo animal. Isso confirma os resultados obtidos, pois os níveis de inclusão de MOS interferiram na conversão alimentar, devido ao aumento das vilosidades intestinais, da densidade de vilos e do comprimento do intestino. Como não ocorreram diferenças significativas no consumo de ração e no ganho de peso, a melhora na conversão alimentar pode ser atribuída ao aumento da área de absorção de nutrientes, resultante da adição de MOS.

Os resultados da contagem de células caliciformes revelaram algumas considerações de grande relevância, considerando que a principal função das células caliciformes é a produção de muco, constituído por glicoproteínas intensamente hidrofílicas, cuja função principal é proteger e lubrificar o revestimento do intestino (Junqueira \& Carneiro, 2008). Quando um epitélio intestinal apresenta grande número de células caliciformes, isso pode ser indicativo de um processo de agressão causado por bactérias, fungos e toxinas, entre outros. Foi observado neste estudo que, embora não haja diferença significativa entre os tratamentos, o aumento no número de células caliciformes $(\mathrm{P}>0,05)$ não correspondeu ao da altura das vilosidades intestinais. Esse fato pode ser um indicativo de que, uma vez a vilosidade estando em estado de integridade, e o MOS atuando na redução de colonização por bactérias enteropatogênicas, o trato gastrointestinal pode estar em equilíbrio, não necessitando para isso de um turnover celular para a regeneração ou integridade do tubo digestório de peixes. Isso confirma os dados obtidos por Resfile et al. (2010), que testaram a resistência de salmões (Salmo salar) desafiados com Caligus elongatus com $2000 \mathrm{mg} \mathrm{MOS} \mathrm{kg-}^{1}$ na dieta e observaram que os peixes tratados com este prebiótico apresentaram morfologia intestinal com maior integridade.
$\mathrm{Na}$ avaliação da integridade da mucosa intestinal após o período de masculinização (Figura 1), observou-se ação prebiótica do MOS sobre a densidade de vilos e altura das vilosidades intestinais, comprovando que sua ação sobre a morfometria intestinal contribui para melhor saúde intestinal, melhorando a utilização dos nutrientes e o desempenho produtivo dos peixes.

\section{Conclusões}

O nível de $0,34 \%$ de mananoligossacarídeos pode ser incluído em dietas para larvas de tilápias-do-nilo durante o período de masculinização para melhorar a morfologia da mucosa intestinal e a conversão alimentar dessa espécie.

\section{Agradecimentos}

Saf do Brasil/Dr. Alfredo de Andrade Navaro; Supra Rações, Catallini, Coamo, Sun Lac, FAFIPAR, Programa de Pós-Graduação em Zootecnia/UEM/PPZ.

\section{Referências}

ALERTS, B.; JOHNSON, A.; LEWIS, J. et al. Biologia molecular da célula. 4.ed. Porto Alegre: Artmed, 2004. 1463p.

BACILA, M. Bioquímica veterinária. 2.ed. São Paulo: Robe, 2003. $583 \mathrm{p}$.

CULJAK, V.; BOGUT, G.; HAS-SHON, E. et al. Effect of Bio-MOS on performance and health of luvenile carp. In: ALLTECCH'S ANNUAL SYMPOSIUM, Lexington: Nutrition and biotechnology in the feed and food industries, 2006. p.153-161.

FURLAN, R.L. Avaliação e uso de pré e probióticos. In: SIMPÓSIO BRASIL SUL DE AVICULTURA, 6., 2005, Chapecó. Anais... Chapecó, 2005. p.58-74.

GIBSON, G.R.; ROBERFROID, M.M. Dietary modulation of the human colonic microbiota: Introducing the concept of prebiotics. Journal of Nutrition, v.125, p.1401-1412, 1995.

GRISDALE-HELlAND, B.; HELlAND, S.J.; GATLIN, D.M. The effects of dietary supplementation with mannanoligosaccharide, fructooligosaccharide or galactooligosaccharide on the growth and feed utilization of Atlantic salmon (Salmo salar). Aquaculture, v.283, n.1-4, p.163-167, 2008.

HAYASHI, C. Breves considerações sobre tilápias. In: RIBEIRO, R.P.; HAYASHI, C; FURUYA, W.M. (Eds). Curso de piscicultura criação racional de tilápias. 1.ed. Maringá: ADUEM, 1995. p.4.

HISANO, H. Levedura desidratada íntegra, autolizada e parede celular como pró-nutrientes para tilápia do Nilo. 2005. 90f. Tese (Doutorado em Zootecnia) - Faculdade de Medicina Veterinária e Zootecnia/Universidade Estadual Paulista, Botucatu.

HISANO, H.; SAMPAIO, F.G.; BARROS, M.M. et al. Composição nutricional e digestibilidade aparente da levedura íntegra, da levedura autolisada e da parede celular pela tilápia-do-Nilo. Ciência Animal Brasileira, v.9, n.1, p.43-49, 2008.

HOUGH, J.S. Biotecnologia de la cerveza y de malta. Zaragoza: Acribia, 1990. 194p.

IJI, P.A.; SAKI, A.A.; TIVEY, D.R. Intestinal structure and function of broiler chickens on diets supplemented with a mannan oligosaccharide. Journal Science Food Agriculture, v.81, p.1186-1192, 2001 . 
JAUNCEY, K.; ROSS, B. A guide to tilapia feeds and feeding. Scotland: University, 1982. $241 \mathrm{p}$.

JUNQUEIRA, L.C.; CARNEIRO, J. Biologia celular. 8.ed. Rio de Janeiro: Guanabara Koogan S.A., 2005. 302p.

JUNQUEIRA, L.C.; CARNEIRO, J. Histologia básica. 11.ed. Rio de Janeiro: Guanabara Koogan S.A., 2008. 524p.

LI, P.; GATLIN III, D.M. Evaluations of brewers yeast (Saccharomyces cerevisiae) as feed supplement for hybrid bass (Morone chrysops $x$ M. saxatilis). Aquaculture, v.219, p.681-692, 2003.

LI, P.; GATLIN III, D.M. Dietary brewers yeast and the prebiotic Grobiotic ${ }^{\mathrm{TM}}$ AE influence growth performance, immune responses and resistance of hybrd bass (Morone chrysops $\mathrm{x}$ M. saxatilis) to Streptococcus iniae infecton. Aquaculture, v.231, p.445-4456, 2004

MARACANAS, J.M. et al. Genotype and environment: A comparative evaluation of four tilápia stocks. Aquaculture, v.150, p.11-24, 1997.

MOREIRA, H.L.M.; VARGAS, L.; RIBEIRO, R.P. et al. Fundamentos da moderna aquicultura. Canoas: ULFLA, 2001. 199p.

NATIONAL RESEARCH COUNCIL - NRC Nutrient requirements of warmwater fishes and shellfishes. Washington: National Academy Press. 1993. 102p.

OSTRENSKY, A.; BORGHETTI, J.R.; SOTO, D. Aquicultura no Brasil: O desafio é crescer. Brasília, 2008. p.276.

REFSTIE, S.; BAEVERFJORD, G.; SEIM, R.R. et al. Effects of dietary yeasty cell wall â-glucans and MOS on performance, gut health, and salmon lice resistance in Atlantic salmon (Salmo salar) fed sunflower and soybean meal. Aquaculture, v.305, p.109-116, 2010.

ROBERTSEN, B.; ENGSTAD, R.; JORGENSEN, J.B.B Glucans as immunostimulants in fish. In: STOLEN, J., FLETCHER, T.C. (Eds.) Modulators of fish immune responses. Fair Haven, NJ: SOS Publications, 1994. p.83-99.

SADO, R.Y.; ALMEIDA BICUDO, A.J.D; CYRINO, J.E.P. Feeding dietary mannan oligosaccharides to juvenile nile tilapis, Oreochromis niloticus, has no effect on hematological parameters and showed decreased feed consumption. Journal of the World Aquaculture Society, v.39, n.6, p.821-826, 2008.

SALZE, G.; MCLEAN, E.; SCHWARZ, M.H. et al. Dietary mannan oligosaccharide enhances salinity tolerance and gut development of larval cobia. Aquaculture, v.274, p.148-152, 2008.

SAKAI, M. Current research status of fish immunostimulants. Aquaculture, v.172, p.63-92, 1999

SCHWARZ, K.S.; FANTA, E.; WERNECK, P.R. et al. Dados preliminares do desenvolvimento e ganho de massa corpórea como consequência da utilização de mananoligossacarídeo na alimentação de juvenis de robalo-peva (Centropomus parallelus) e sua relação com as características teciduais. Curitiba: UFPR, 2005. Disponível em: $<\mathrm{http} / / 25$ pgbiocel.bio.ufpr ResumosPUB/0049.html>. Acesso em: 3 mar. 2006.

SCHWARZ, K.S.; FURUYA, W.M.; LIMA, A.G.P. et al. Mananoligossacarídeo em dieta para juvenis de carpa capim (Ctenopharyngodon idella). In: CONGRESSO INTERNACIONAL SOBRE USO DA LEVEDURA NA ALIMENTAÇÃO ANIMAL/ CBNA, 1., 2009, Campinas. Anais... Campinas, 2009. p.185-186.

SCHWARZ, K.S.; FURUYA, W.M; NATALI, M.R.M. et al. Mananoligossacarídeo em dietas para juvenis de tilapia do Nilo. Acta Scientarum.Animal Sciences, v.32, n.2, p.197-203, 2010.

SILVA, D.J.; QUEIROZ, C. Análise de alimentos: métodos químicos e biológicos. Viçosa, MG: Universidade Federal de Viçosa, 2002. 235p.

SILVA, D.J.; QUEIROZ, A.C. Análise de alimentos: métodos químicos e biológicos. 3.ed. Viçosa, MG: Universidade Federal de Viçosa, 2006. 235p.

SPPRING, P. Yeast's secret wear on aids animal production. Feed Mix, v.22, p.32, 2001

STAYKOV, Y.; DENEV, S.; SPRING, P. Influence of dietary mannan oligosaccharide (Bio-MOS) on e growth rate and immune of common carp (Cyprinus carpio L.). In: HOWELL, B.; FLOS R. (Eds.) Lessons from the past to optimize the future. Tronheim, Norway: European Aquaculture Society, 2005. p.431-432. (Special Publication, 35)

STAYKOV, Y.; SPRING, P.; SWEETMAN, J. Effect of a mannan oligosaccharide on the growth performance and immune status of rainbow trout (Oncorhynchus mykiss). Aquaculture International, v.15, p.153-161, 2007.

TACHIBANA, L.; CASTAGNOLI, N.; PEZZATO, L.E. et al Desempenho de diferentes linhagens de tilápia do Nilo (Oreochromis niloticus) na fase de reversão sexual. Acta Scientiarum Animal Sciences, v.26, n.3, p.305-311, 2004

UNIVERSIDADE FEDERAL DE VICYOSA - UFV. SAEG - Sistema de análises estatísticas e genéticas. Versão 8.0 Viçosa, MG: 2000. (CD-ROM).

VERA CRUZ, E.M.; MAIR, G.C. Conditions for effective androgen sex reversal in Oreochromis niloticus. Aquaculture, v.122, p.27-248, 1994

YILMAZ, E.; GENC, M.A.; GENC, E. Effects of dietary mannan oligosaccharides on growth, body composition, and intestine and liver histology of rainbow trout, Oncorhynchus mykiss. Israeli Journal of Aquaculture, v.59, p.182-188, 2007. 